\title{
Delayed deterioration in patients with traumatic frontal contusions
}

\author{
P F STATHAM, R A JOHNSTON, P MACPHERSON \\ From the Departments of Neurosurgery and Neuroradiology, Institute of Neurological Sciences, Southern \\ General Hospital, Glasgow, Scotland, UK
}

SUMMARY The clinical course of 18 head injured patients in whom CT had shown frontal contusions without diffuse brain injury or intracranial haematoma was reviewed. All 10 patients with unilateral frontal contusion made a good recovery. Only two of five patients with limited bilateral frontal contusions made a good recovery. Two of three patients with extensive bilateral frontal contusions deteriorated more than 24 hours after injury, and one died. Delayed deterioration is an important complication of extensive traumatic bifrontal contusions.

Cerebral cortical contusions are the most common computed tomographic (CT) finding in head injured patients, but few need operative treatment. Nevertheless in $2 \%$ of fatal head injuries only contusions are found at necropsy. ${ }^{1}$ A recent case highlighted how rapidly conscious level can deteriorate and stimulated us to review retrospectively a series of patients with frontal contusions. Our aims were to discover how often deterioration occurred, how it affected outcome, and if patients at risk could be recognised at the time of initial CT.

\section{Case report}

A 29 year old man was assaulted whilst drunk. He sustained a left periorbital haematoma, an occipital laceration and an occipital fracture, and was admitted to hospital. He was initially unconscious but improved over 8 hours to become alert and orientated. Twelve hours after injury he was discharged home. Twenty four hours later his level of consciousness deteriorated, from mild disorientation into coma. He was readmitted to hospital within 6 hours, and referred to this unit. On admission he was flexing to pain, and uttering inappropriate words, but was not opening his eyes. His right pupil was fixed and dilated. CT was performed as an emergency, and showed extensive bilateral subfrontal contusions, most severe on the left side, and smaller temporal contusions (fig 1). A left frontotemporal craniotomy was performed, and the left frontal and temporal contusions were evacuated. A subdural intracranial pressure (ICP) monitor was used to record post operative intracranial pressure,

Address for reprint requests: Mr P F Statham, Department of Neurosurgery, Southern General Hospital, Glasgow G51 4TF, UK

Received 29 April 1988 and in revised form 1 October 1988. Accepted 5 November 1988 which rose above $30 \mathrm{~mm} \mathrm{Hg}$ within 4 hours. His condition deteriorated despite intensive care, and he died 5 days after his injury.

Post mortem examination showed bifrontal and bitemporal haemorrhagic contusions, with bilateral tentorial herniation and haemorrhagic infarction in the region of the left posterior cerebral artery. Haemorrhagic necrosis of the left parahippocampal gyrus had occurred, an indication of

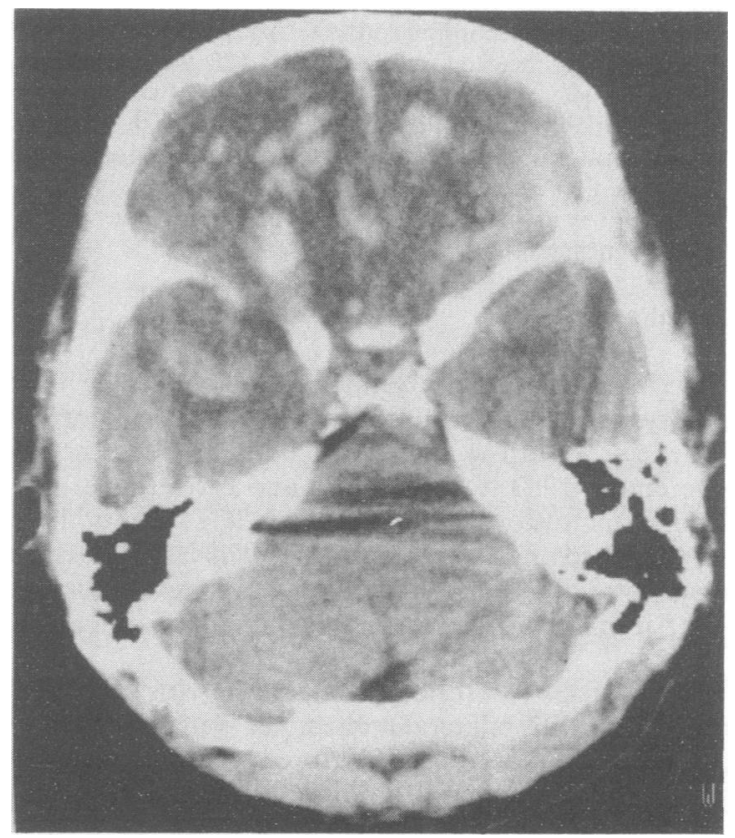

Fig 1 Admission CT showing bilateral frontal and temporal contusions. 
left parahippocampal gyrus had occurred, an indication of raised intracranial pressure during life. ${ }^{2}$ There was no extracranial cause of deterioration, and no other intracranial complication.

\section{Patients and methods}

As part of a study of CT in head injury, all patients admitted to this Regional Neurosurgical Unit between 1 January and 31 December 1983 had their CT scans analysed by a neuroradiologist. The results were separated into diagnostic categories, which included contusions, haematomas and diffuse injury, and were stored on computer. This database was used to identify all patients with frontal contusions. These were defined as regions of mixed high and low attenuation (or occasionally low attenuation only) in the frontal lobes. Where a discreet region of high attenuation predominated the lesion was classified as a haematoma. In any case where there was doubt between the two entities the lesion was invariably classified as a haematoma. The case records of those patients with frontal contusions, but without other major lesions or diffuse brain injury, were retrospectively reviewed.

The following features were recorded: patients' age and sex, time of first assessment after injury, Glasgow Coma Score $^{3}$ on admission to this unit, pupil reactions, eye movements, focal signs, seizures, hypoxia or hypotension, extracranial injuries, and skull fracture. Medical or operative treatment was recorded, with details of the nature of the operation and indications. The cause and time of any clinical deterioration was documented.

Outcome within $\mathbf{4 8}$ hours of admission, on discharge from the Neurosurgical Unit, and 6 months from injury, were noted, the latter according to the Glasgow Outcome Scale. ${ }^{4}$ The following features on the initial CT were reviewed; contusion site (unilateral, unilateral with other sites, or bilateral), contusion depth (limited or extensive), effacement of the third ventricle or basal cisterns, and midline shift. Extensive contusions were defined as those involving both frontal lobes as far posteriorly as the anterior horn of the lateral ventricles.

\section{Results}

Five hundred and twenty nine patients with acute head injury were admitted to the Institute of Neurological Sciences, Glasgow between 1 January and the 31 December 1983. One hundred and three $(20 \%)$ had frontal contusions demonstrated by CT. Eighty three of these had another major intracranial lesion, such as haematoma. Of the remaining 20 , two were unavailable for follow up. This review is therefore based on 18 cases.

Two groups were identified; those with unilateral and those with bilateral contusions. Ten patients had unilateral frontal contusions, three of whom had other contusions; bitemporal, temporal, and parietal respectively. The remaining eight had bilateral frontal contusions, and in three these were extensive. Fifteen patients were male, and three were female, aged 3 to 64

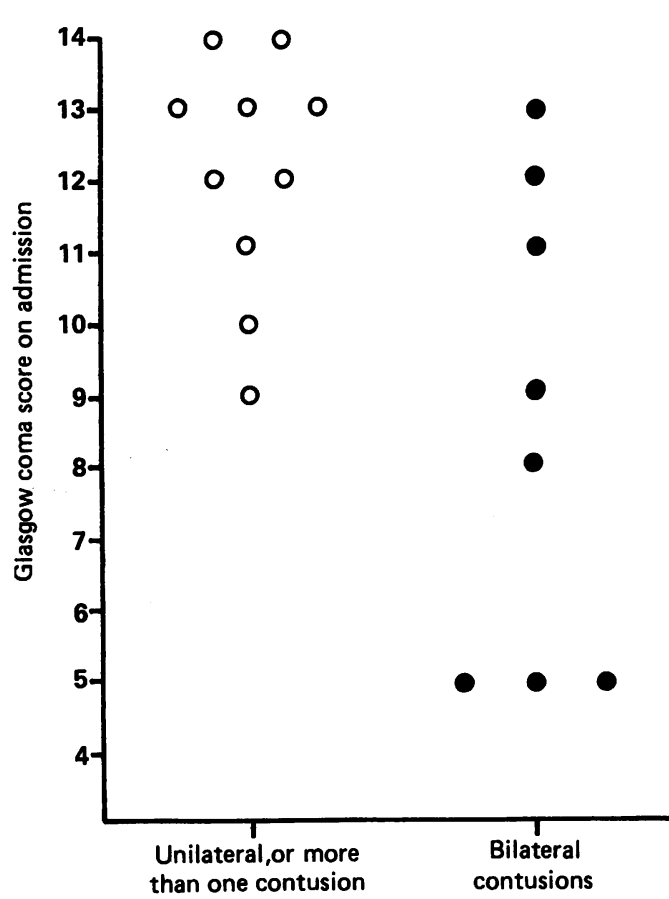

Fig 2 Glasgow Coma Score of patients admitted with frontal contusions.

years (mean 36 years). Twelve had a skull fracture ( occipital, 4 frontal, 4 elsewhere). Glasgow Coma scores at the time of CT were between five and 14 (ffi 2). There was no statistical difference between the two groups ( $p>0.01$ Wilcoxon two sample test).

Sixteen of the 18 patients improved during their stay in this Unit. Two patients deteriorated. Both had extensive bifrontal contusions on CT at admission. The first improved initially, from a GCS of 11 to a GCS of 12, but 3 days after injury deteriorated over 3 hours. At this point he was in coma, localising a painful stimulus, but neither opening his eyes nor making any verbal response. Repeat CT showed the development of a right frontal intracerebral haematoma (fig 3). Surgical evacuation of the haematoma and contusion led to a good recovery. This was the only patient of either group who had an intracranial operation.

The second patient had extensive bifrontal contusions with diastasis of the sagittal suture. His coma score on admission was five, but improved to nine within $\mathbf{4 8}$ hours. However on the ninth day following injury he progressively deteriorated and died. Necropsy confirmed bilateral subfrontal contusions and a left sided tentorial hernia, parahippocampal necrosis, and a haemorrhagic infarct in the territory of 

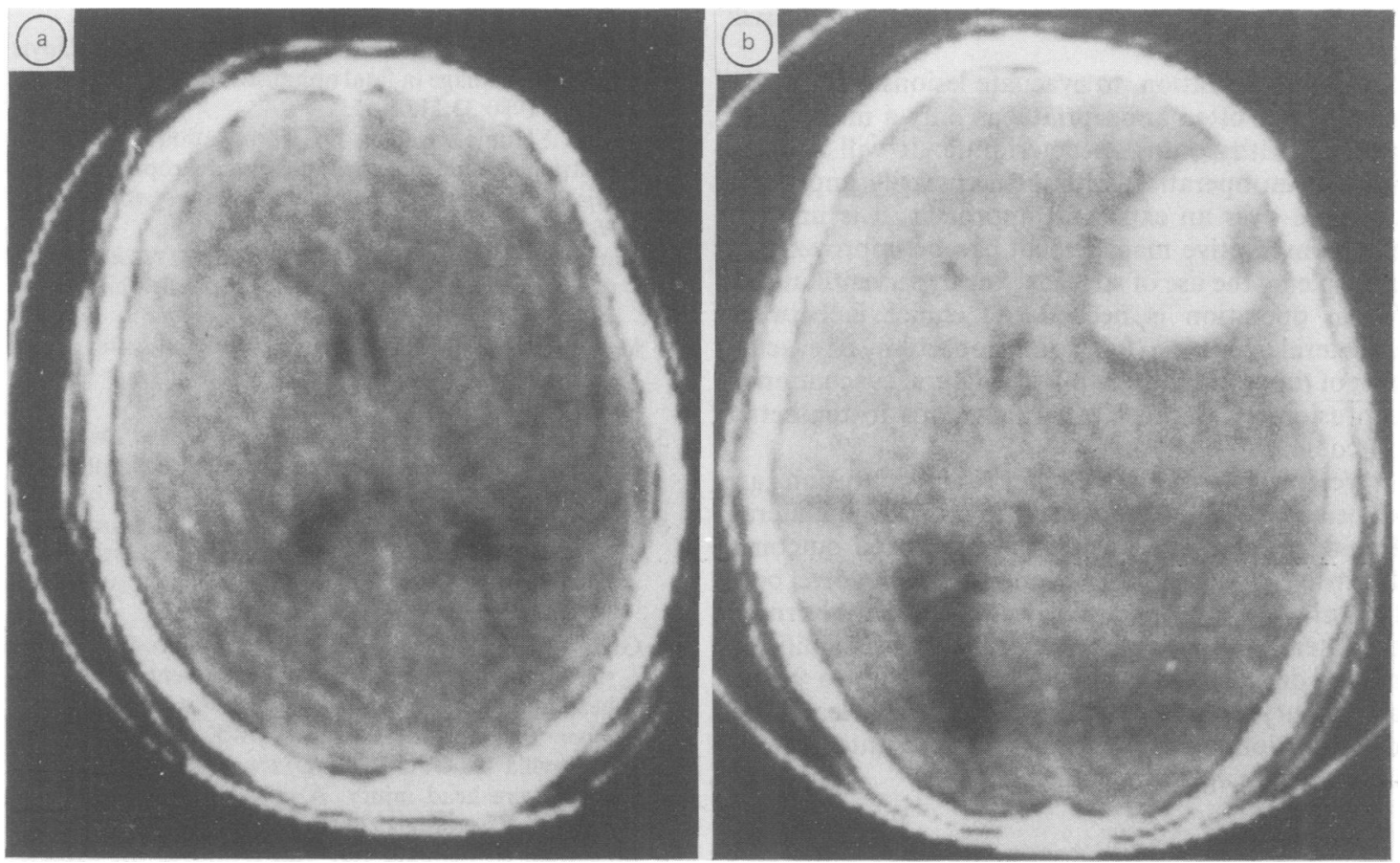

Fig 3 CT on admission (a) and after deterioration (b) showing the development of a large right frontal intracerebral haematoma.

the left posterior cerebral artery. There was no lateral shift, and no other cause for deterioration.

During the 6 months after injury all 10 patients with unilateral frontal contusions made a good recovery, although one died 5 months after injury from an intracerebral haemorrhage, confirmed at necropsy. Of those with limited bilateral frontal contusions, one patient was severely disabled, two were moderately disabled and two made a good recovery. Of three patients with extensive bilateral frontal contusions, one died, another deteriorated but made a good recovery after operation (both described above), and the third had an ICP of $30 \mathrm{~mm} \mathrm{Hg}$, but made a good recovery without operation.

No other feature of the initial CT scan was shown to predict deterioration.

\section{Discussion}

The management of a patient with marked frontal cerebral contusions can be more difficult than a patient with a major intracranial haematoma. Operation will become necessary in about $50 \%$ of patients with intracranial haematoma, even when the patient is clinically stable; moreover the need for operation can be predicted by ICP monitoring, and is most likely in the first 3 days after injury. ${ }^{5}$ The finding of contusion only leads to much less vigilance, because the risk of deterioration is thought to be less. There are no previous data about how often deterioration occurs, nor about which cases are most at risk. Space occupying lesions in the frontal lobes, either from trauma or tumour, may declare abruptly, as our cases show. This is because shift is anterioposterior rather than lateral, so that the classical "warning" lateralising signs develop very much later.

Of 18 patients whose CT showed frontal contusion, the 10 who had unilateral contusions all made a good recovery. Four of five patients with limited bilateral contusions made a moderate or good recovery. Two of three patients with extensive bilateral lesions deteriorated. This occurred as late as $\mathbf{9}$ days after injury, and was due to raised intracranial pressure, produced by a combination of mass effect from swelling around the contusion, and in one case, the development of a sizeable delayed haematoma in the contused area.

The value of ICP monitoring in patients with contusions is not as clear as in patients with traumatic intracranial haematoma. The patients most likely to develop raised ICP are those with extensive bifrontal contusions, but an ICP of less than $30 \mathrm{~mm} \mathrm{Hg}$ in the first few days after injury does not exclude later deterioration. Therefore if ICP monitoring is used, it 
may be needed for several days. This increases the risk of complications. ${ }^{6}$

Elective operation, to evacuate lesions seen on CT scan, is not often appropriate, as only a minority of patients with contusion deteriorate. It will add the risks of an operation without necessarily improving outcome over an expectant approach. It is unlikely that conservative management can be improved, for example by the use of steroids ${ }^{7-9}$ or hyperventilation..$^{10}$ When operation is needed the choice is between unilateral operation (full frontal lobectomy or evacuation of the worst contusion), or bilateral evacuation of contusions. It is not clear which leads to the better outcome.

We conclude that traumatic frontal contusions are associated with a good outcome when unilateral. Limited bilateral contusions have a mixed outcome. Extensive bifrontal contusions, which involve both frontal lobes as far posteriorly as the anterior horns of the lateral ventricles, are associated with significant risk of deterioration, which may occur up to 9 days after injury. More knowledge of the factors leading to deterioration, and how it might be prevented, would improve management.

We thank Professor G M Teasdale for his helpful advice in preparing this paper.

\section{References}

1 Adams J Hume, Graham DI, Scott G, Parker LS, Doyle D. Brain damage in fatal nonmissile head injury. J Clin Pathol 1980;33:1132-45.

2 Adams J Hume, Graham DI. The relationship between ventricular fluid pressure and the neuropathology of raised intracranial pressure. Neuropathol Appl Neurobiol 1976;2:323-32.

3 Jennett B, Teasdale G, eds. Management of Head Injuries, Contemporary Neurology Series. Philadelphia: Davis 1981;1:77-93.

4 Jennett B, Bond M. Assessment of outcome after severe brain damage. A practical scale. Lancet 1975; i: 480-484.

5 Galbraith S, Teasdale G. Predicting the need for operation in the patient with an occult traumatic intracranial haematoma. $J$ Neurosurg 1981;55:75-81.

6 Narayan RK, Kishore PS, Becker DP, et al. Intracranial Pressure: To monitor or not to monitor? A review of our experience with severe head injury. $J$ Neurosurg 1982;56:650-9.

7 Gudeman SK, Miller JD, Becker DP. Failure of high dose steroid therapy to influence ICP in patients with severe head injury. $J$ Neurosurg 1979;51:301-6.

8 Cooper PR, Moody S, Clark WK, Kirkpatrick J, Maravilla K, Gould AL, Drane W. Dexamethasone and severe head injury. A prospective double blind study. J Neurosurg 1979;51:307-16.

9 Brackman R, Schouta HJ, Van Dishoeck M, Minderhoud JM. Megadose steroids in severe head injury. $J$ Neurosurg 1983;58:326-30.

10 Jennett B, Teasdale G, Fry J, et al. Treatment for severe head injury. J Neurol Neurosurg Psychiatry 43:289-95. 